\title{
Modelling weathering, climate and biogeochemistry over Phanerozoic time
}

BENJAMIN J. W. MILLS ${ }^{1}$, YANNICK DONNADIEU ${ }^{2}$ AND YVES GODDÉRIS ${ }^{3}$

${ }^{1}$ School of Earth and Environment, University of Leeds

${ }^{2}$ CEREGE, Aix Marseille University

${ }^{3}$ Géosciences Environnement Toulouse

Presenting Author: b.mills@leeds.ac.uk

It is important to understand how Earth's surface conditions have changed over geological timescales and what has driven these changes. Much of this understanding comes from combining geological and geochemical data with global biogeochemical models, but designing and running computer models over these vast timeframes poses a serious challenge. Traditionally one must choose between running a climateenabled model for sets of shorter-term scenarios or running a dimensionless 'box' model for long periods, but with a poor representation of climate and surface processes. In this presentation we introduce a method to run a climate-enabled biogeochemical model over Phanerozoic timescales: which we term Spatial Continuous Integration (SCION). This approach combines two key methods in the literature, which form the basis of the 'GEOCLIM' and 'COPSE' models respectively, and the resulting model is able to compute whole-Phanerozoic linked climate and biogeochemistry, as well as outputting geochemical isotope tracers to aid in hypothesis testing. This new model differs from GEOCLIM because it is able to run continuously over the whole Phanerozoic, and it differs from COPSE by having a spatial representation of climate and continental processes.

Spatial representation of climate allows for detailed approximations for surface weathering processes through changes in temperature, erosion and runoff, and the SCION predictions for atmospheric $\mathrm{CO}_{2}$ variations over Phanerozoic time show better agreement with data than predictions from nondimensional models. Despite this, several inconsistencies remain with the geological record, most notably the inability of the model to reproduce transient late Ordovician cooling, and general over-estimation of $\mathrm{CO}_{2}$ levels during the Paleozoic. These discrepancies may be addressed in future work by including a scheme for positioning of highly-weatherable volcanic terranes, and by better capturing the behaviour of the terrestrial biosphere. The model approach we outline here is ideally suited for expansion to include these aspects. 